\title{
Buprenorphine- and Methadone Maintenance Treatment: Influence on Aspects of Cognitive and Memory Performance
}

\author{
S.M. Giacomuzzi ${ }^{*}, 1,2$, C. Thill ${ }^{2}$, Y. Riemer ${ }^{1}$, K. Garber $^{3}$ and M. Ertl ${ }^{1}$ \\ ${ }^{1}$ Medical University Innsbruck, Innsbruck General Hospital, Innsbruck, Austria \\ ${ }^{2}$ Institute of Psychology, University Innsbruck, Innsbruck, Austria \\ ${ }^{3}$ Free University of Bozen, Bolzano, Italy
}

\begin{abstract}
Our randomized study investigated neurocognitive performance, such as working memory, verbal and figural memory, executive functions and psychomotor speed of opioid-dependent patients within a permanent maintenance treatment programme. Our results indicate that buprenorphine preserve cognitive functions better than methadone, at least when benzodiazepine comedication is used. Buprenorphine-treated patients showed significantly better results compared to methadone patients in the verbal memory performance, and this even for the verbal list learning $(p=0.007)$ as for the delayed recall of the words $(p=0.012)$. Furthermore the buprenorphine maintained persons showed statistically significant the more favourable scores in the working memory performance for figural material (digit symbol test, HAWIE-R) $(p=0.021)$. Our results indicate a better cognitive performance also after a longer substitution time and results cannot easily be attributed to an "only" transient opioid switching effect. Continued research is still needed to confirm the less impairment on cognitive functions.
\end{abstract}

\section{BACKGROUND}

Both buprenorphine- and methadone-treated opioiddependent patients often show cognitive deficits in attention, working memory, and verbal memory. However, only a few studies have compared these patient groups with each other during permanent maintenance treatment [1-8]. It was concluded that the risk of neuropsychological impairment is greater in opiate abusers (Davis et al. 2002), and that recovery may occur during abstinence [9]. In a randomized study Soyka et al. (2005) compared both treatments in drug dependent patients, where buprenorphine produced partially less impairment on cognitive functions in some of the subtests of the psychomotor battery than methadone [10].

Therefore, we investigated neurocognitive performance, such as working memory, verbal and figural memory, executive functions and psychomotor speed of opioid-dependent patients within a permanent maintenance treatment programme in a randomized study.

\section{METHODS}

We performed a randomized clinical trail to 37 drugdependent patients under either buprenorphine or methadone treatment. The sample included 24 methadone-, and $13 \mathrm{bu}-$ prenorphine -treated patients. All patients who agreed to participate, had been informed before starting with the tests, about the duration of the examination and the topic of the study. Therefore, all volunteers, who accepted to participate, accomplished all the tasks in the test battery. No dropouts of treatment were noticed during the examination period. As all

*Address correspondence to this author at the Medical University Innsbruck, University Department of Psychiatry, Anichstr. 35, 6020 Innsbruck, Austria; Tel: +43 512-504-24750; Fax: +43 512-504-24764;

E-mail: salvatore.giacomuzzi@i-med.ac.at patients were already in a normal maintenance programme there was no need for ethical approval. Furthermore, the participants were not compensated for this study. The only reward for participation was a feedback over their test results.

The methadone group was demographically similar to the buprenorphine group and balanced for sex (methadone $\mathrm{m}=$ $15 ; \mathrm{f}=9$; buprenorphine $\mathrm{m}=7 ; \mathrm{f}=6(\mathrm{p}=0.730))$ and age (methadone $\mathrm{Md}=31 \mathrm{a}$; IQR = 25.3-42.0; buprenorphine $\mathrm{Md}=$ $33 \mathrm{a} ; \mathrm{IQR}=24.5-38.5(\mathrm{p}=0.937))$. The study took place in the "Drug ambulance Innsbruck" during a period of 8 weeks. The study-participants with opioid dependence were volunteers and had been recruited randomly, simply by asking those patients who were present at that period of time in the "addiction ambulance". To exclude participants with current uncontrolled polysubstance abuse or acute alcohol abuse, and to access long-term effects on the cognitive performance of the given medications, we recruited only those patients who conformed to these requirements and who showed a sufficient compliance. All patients who agreed to participates, had been informed about the duration of the examination and the topic of the study. In this naturalistic study, all participants received their prescribed psychoactive medications according to their clinical dose regimen, before starting with the tests. To determine the additional consumption of psychotropic substances of the participants, a self-developed questionnaire was used to collect this data. Benzodiazepine codependence, recent use, and comedication were also common in both patient groups.

Analysis of variance was used to study the overall group effect in each cognitive test. In order to investigate if group differences were due to differences in other factors between the groups, we set the variables sex, age and duration of opioid-addiction as a covariate to the measures. All statistically significant group differences, except the result in the subtest "immediate recall" (VLMT), remained significant after adjusting for the covariate. 
Pair-wise group comparisons were made, when appropriate. We applied the Verbal Learning and Memory Test (VLMT), the Rey Complex Figure Test (RCFT), the Trail Making Test A \& B (TMT), the HAWIE-R (digit symbol test) and the Regensburger Word Fluency - Test (RWT). To control the potential depression-related effects and the influence of pre-morbid intelligence function on neuropsychological performance we used the Beck Depression Inventory (BDI) and the the Mehrfachwahl-Wortschatz-Intelligence Test (MWT-B).

\section{RESULTS}

Time of opioid dependence (methadone $\mathrm{Md}=12 \mathrm{a} ; \mathrm{IQR}=$ $5,1-23,8 \mathrm{a}$; buprenorphine $\mathrm{MD}=6 \mathrm{a} ; \mathrm{IQR}=3.0-17.5 \mathrm{a}(\mathrm{p}=$ 0.263 ) and time of substitution treatment (methadone $\mathrm{MD}=$ 2.3a; IQR = 0.8-11.5a; buprenorphine $\mathrm{MD}=2.3 \mathrm{a}$; IQR $=1.5$ $4.6 \mathrm{a}(\mathrm{p}=0.845)$ between the two groups were not significantly different. Additional consumption of psychotropic substances between the two maintenance groups also showed no significant differences $(\mathrm{p}=0.739) .11$ of the 24 patients of the methadone group $(45,8 \%)$ and 5 of the 13 patients of the buprenorphine group $(38,5 \%)$ confirmed to consume at least one additional psychotropic substance $(\mathrm{p}=0.739)$. In the methadone group, morphine and heroin were the main opioids of abuse during the recent month $(109,2 \%$ of the answers), followed by cannabioid $(72,7 \%)$ and benzodiazepine $(27,3 \%)$. The most given answers in the buprenorphine group come under the cannabioid category $(60 \%)$, followed by heroine $(20 \%)$ and benzodiazepine $(20 \%)$. The mean dose in the methadone group was $65 \mathrm{mg}(\mathrm{IQR}=50-90 \mathrm{mg})$ ) of methadone and $4 \mathrm{mg}$ (IQR $=2-10 \mathrm{mg}$ ) of buprenorphine in the other group. By calculating the equivalent dosage for the buprenorphine group the factor 4, 1666 was used, which resulted on a mean dose of $16,7 \mathrm{mg}$ for the buprenorphine- treated patients. This means that the daily dosage of methadone was "high" significantly higher $(p<0.001)$ than this once of the buprenorphine treated patients. There was no difference between the groups on premorbid functioning $(p=0.888)$ and the depressive scores $(p=0.962)$. There was also no difference between the different substituted patients found for the cognitive performance in the figural memory (RCFT), psychomotor speed (Trail A) and the executive functions (word-fluency (RWT), cognitive flexibility (Trail B)). Apart from those findings, buprenorphine-treated patients, as a group, had significantly better results compared to methadone -treated patients in the verbal memory performance (VLMT), and this even for the verbal list learning $(p=0.007)$ as for the delayed recall of the words $(p=0.012)$. Furthermore, the buprenorphine maintained persons showed statistically significant the more favorable scores in the working memory performance for figural material (digit symbol test, HAWIE-R) $(\mathrm{p}=0.021)$.

\section{CONCLUSION}

Several psychoactive abused medications were used nearly similarly in both of the study groups. Thus, this study further shows that in clinical samples, in which recent benzodiazepine use and benzodiazepine comedication as well as other psychoactive medications are common, methadone-treated patients have more cognitive deficits than buprenorphine treated patients. Buprenorphine may preserve cognitive function better than methadone, at least when benzodiazepine comedication is used. Partly in contrast to the hypotheses of Rapeli et al. [1] buprenorphine patients also showed the better cognitive performances after a longer substitution time and results cannot be easily attributed to an "only" transient opioid switching effect.

But this conclusion is somewhat limited by the small sample size and the unequal size over the two groups. Furthermore cognitive differences between the patient groups may relate to differences in the daily dosages, which were significantly higher in the methadone group than in the buprenorphine group. Continued research is needed to confirm the less impairment on cognitive functions, when drug-dependent patients are treated with buprenorphine than with methadone.

\section{REFERENCES}

[1] Rapeli P, Fabritius C, Alho H, Salaspuro M, Wahlbeck K, Kalska H. Methadone $v s$ buprenorphine/naloxone during early opioid substitution treatment: a naturalistic comparison of cognitive performance relative to healthy controls. BMC Clin Pharmacol 2007; 7: 5.

[2] Giacomuzzi SM, Ertl M, Vigl A, et al. Driving capacity of patients treated with methadone and slow-release oral morphine. Addiction 2005; 100(7): 1027.

[3] Comer SD, Walker EA, Collins ED. Buprenorphine/naloxone reduces the reinforcing and subjective effects of heroin in heroindependent volunteers. Psychopharmacology (Berl) 2005; 181: 66475 .

[4] Harris DS, Mendelson JE, Lin ET, Upton RA, Jones RT. Pharmacokinetics and subjective effects of sublingual buprenorphine, alone or in combination with naloxone - Lack of dose proportionality. Clin Pharmacokinet 2004; 43: 329-40.

[5] Soyka M, Horak M, Dittert S, Kagerer S. Less driving impairment on buprenorphine than methadone in drug-dependent patients? J Neuropsychiatry Clin Neurosci 2001; $13: 527-8$.

[6] Pirastu R, Fais R, Messina M, et al. Impaired decision-making in opiate-dependent subjects: Effect of pharmacological therapies. Drug Alcohol Depend 2006; 83: 163-8

[7] Streel E, Antoniali V, Campanella S, et al. Evaluation of cognitive functioning in 101 patients before opiate detoxification: Implications in setting up therapeutic strategies. J Opioid Manag 2005; 1: 49-53.

[8] Darke S, Sims J, McDonald S, Wickes W. Cognitive impairment among methadone maintenance patients. Addiction 2000; 95: 687-95.

[9] Davis PE, Liddiard H, McMillan TM. Neuropsychological deficits and opiate abuse. Drug Alcohol Depend 2002; 67: 105-8.

[10] Soyka M, Hock B, Kagerer S, Lehnert R, Limmer C, Kuefner H. Less impairment on one portion of a driving-relevant psychomotor battery in buprenorphine-maintained than in methadone-maintained patients: Results of a randomized clinical trial. J Clin Psychopharmacol 2005; 25: 490-3. 